\title{
この出版物は未入力です。 図書室でご覧下さい。
}

This publication is not inputted,

please come and look at the Library. 